\title{
Nanoscale calcium salt-based formulations as potential therapeutics for osteoporosis
}

\author{
Khandmaa Dashnyam ${ }^{a, b, e,{ }^{*},}$ Oyunchimeg Bayaraa ${ }^{a, b, e}, N^{2}$ din Mandakhbayara,b, \\ Jeong-Hui Park ${ }^{a, b}$, Jung-Hwan Lee ${ }^{a, c}$, Tae-Su Jang ${ }^{d}$, Khurelbaatar Luvsane, \\ Hae-Won Kimª,b,c,
}

alnstitute of Tissue Regeneration Engineering (ITREN), Dankook University, Cheonan 330-714, South Korea

bDepartment of Nanobiomedical Science \& BK21 PLUS Global Research Center for Regenerative Medicine, Dankook University, Cheonan 330-714, South Korea

'Department of Biomaterials Science, College of Dentistry, Dankook University, Cheonan 330-714, South Korea

dDepartment of Pre-medi., College of Medicine, Dankook University, Cheonan 31116, Republic of Korea

eDrug Research Institute, Mongolian University of Pharmaceutical Science, 14250, Mongolia

*Co-corresponding authors: KD (khandmaa@naver.com), HWK (kimhw@dku.edu), KL (khurelbaatar@monos.mn)

Khandmaa Dashnyam, Ph.D., Researcher, Institute of Tissue Regeneration Engineering (ITREN), Dankook University, Cheonan 330-714, South Korea, Tel: +82 41550 3081; Fax: +82 41559 7839; E-mail: khandmaa@naver.com

Hae-Won Kim, Ph.D., Professor, Institute of Tissue Regeneration Engineering (ITREN), Dankook University, Cheonan, South Korea,

Tel: +82 41550 3081; Fax: +82 41559 7839; E-mail: kimhw@dku.edu

Khurelbaatar Luvsan, Ph.D., Professor, Drug Research Institute, Mongolian University of Pharmaceutical Science, Ulaanbaatar, Mongolia Tel: + 976-11-77001471; E-mail: khurelbaatar@monos.mn 


\section{Supplementary information}

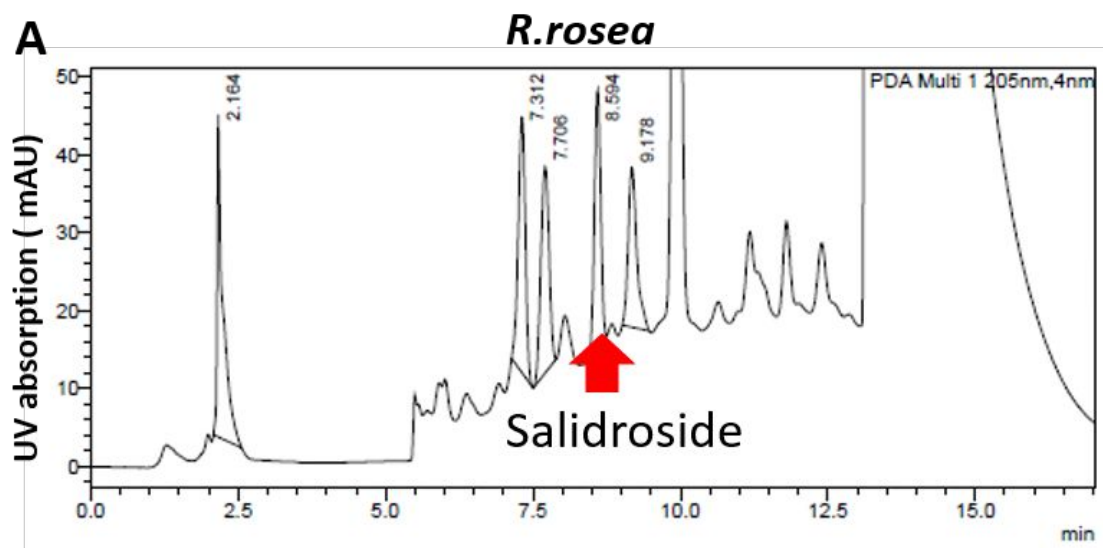

B

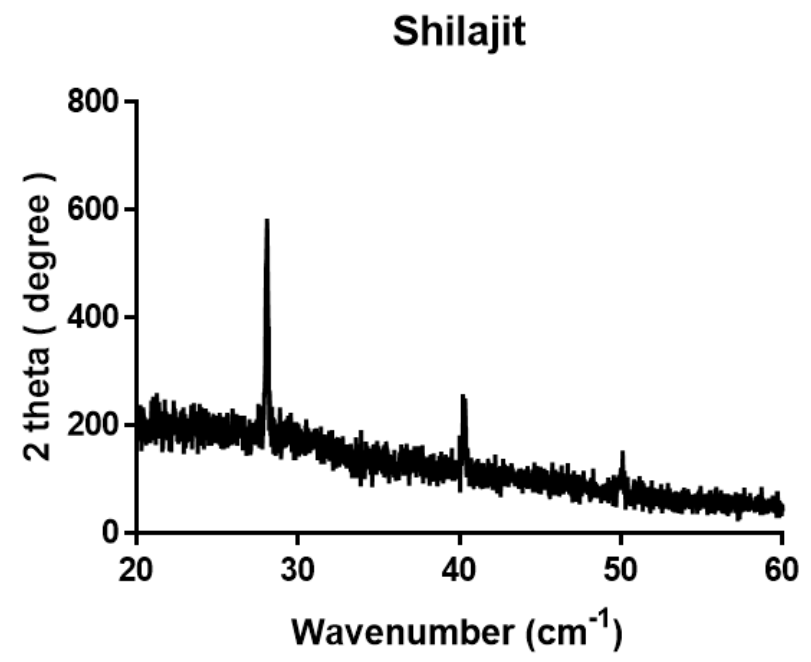

Figure.S2-S1. Characterization of $\boldsymbol{R}$.rosea and Shilajit. A) HPLC detection of active components (salidroside, etc.) in R. rosea. B) XRD conducted to confirm fulvic acid and humic acid in Shilajit. 


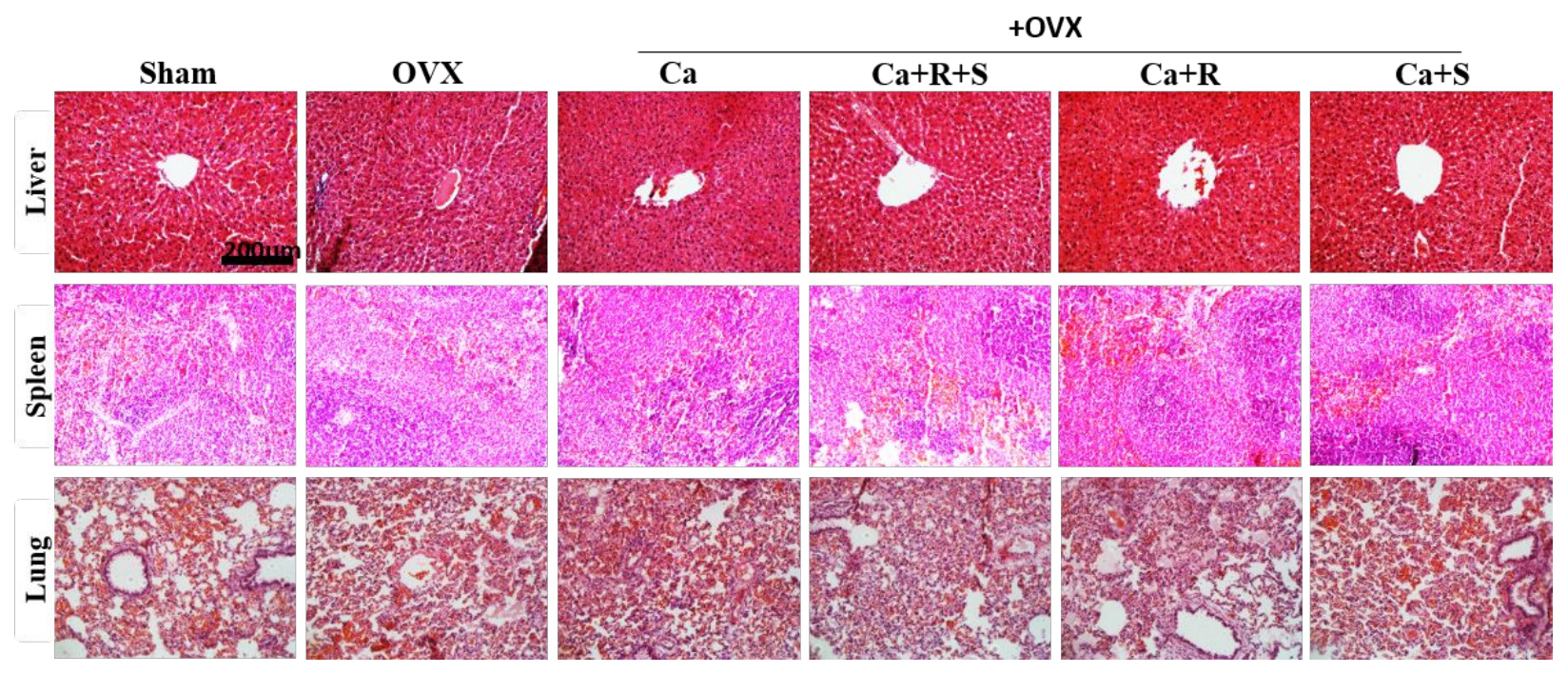

Figure.S3-S2. Organ toxicity. H\&E staining showed no toxicity in the internal organs after oral administration of the drug formulations for 45 days. Scale bar: $200 \mu \mathrm{m}$.

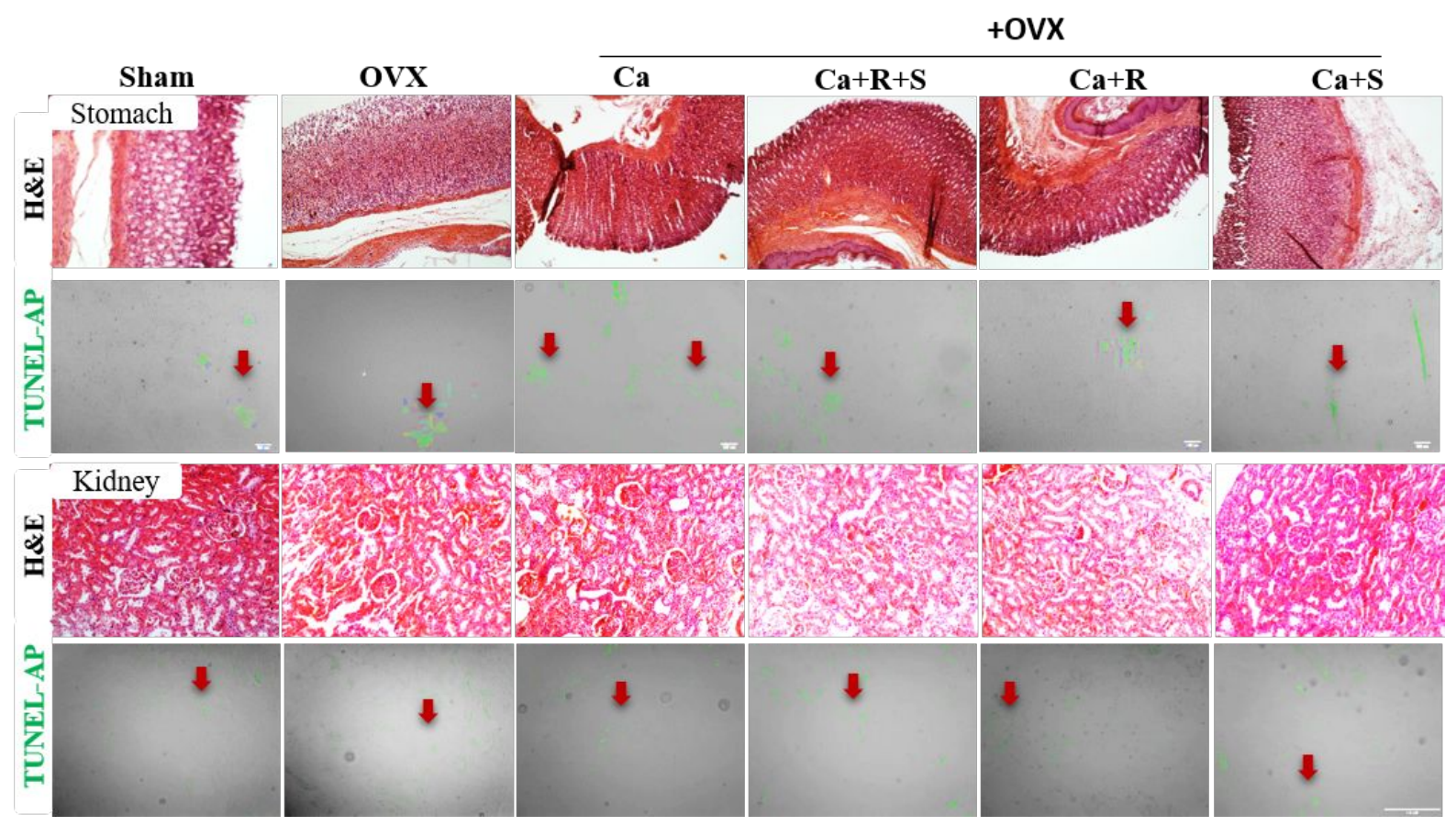

Figure.S3-S3. Biocompatibility of the drug formulations in the stomach and kidney (H\&E and TUNEL-AP staining). All groups showed similar indications. 

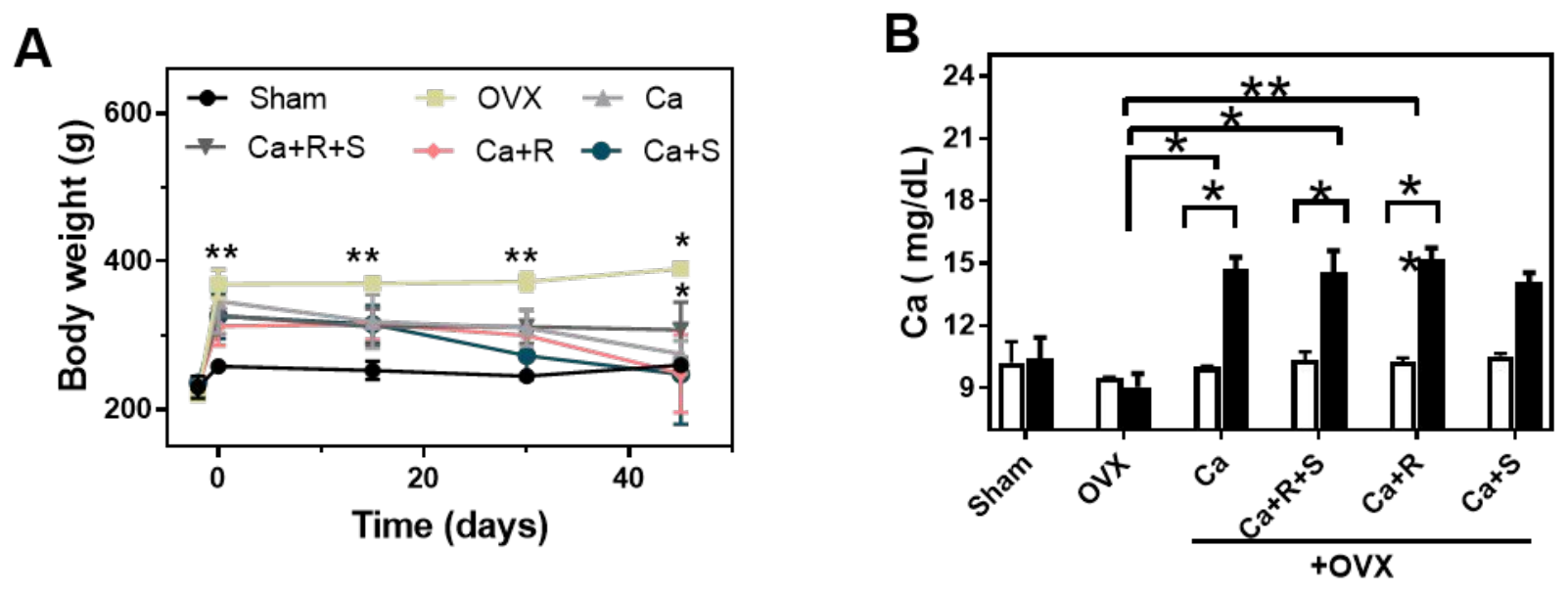

Figure.S4-S4. (A) Body weight measured before and after OVX surgery. B) Blood calcium level measured. $\left(p^{*}<0.05 \&{ }^{* *} p<0.01\right)$.

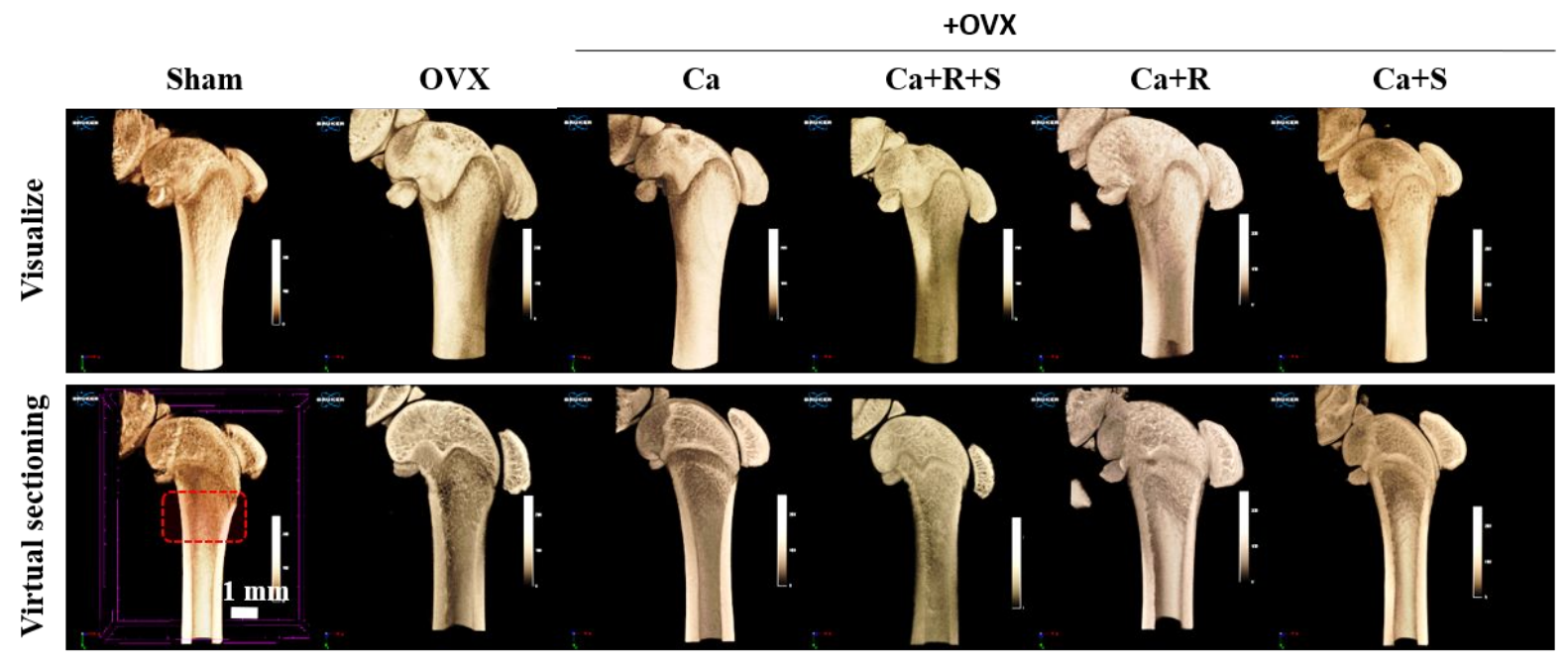

Figure.S4-S5. Femur bone visualized and vertical sectioned images. Scale bar = $1 \mathrm{~mm}$ 